\title{
On the origin of mesoscale TIDs at midlatitudes
}

\author{
M. C. Kelley \\ School of Electrical and Computer Engineering, Cornell University, Ithaca, NY, USA
}

Received: 24 March 2010 - Revised: 3 December 2010 - Accepted: 13 January 2011 - Published: 21 February 2011

\begin{abstract}
A recent breakthrough experiment by Ogawa et al. (2009) showed that Mesoscale Traveling Ionospheric Disturbances (MSTIDs), a common phenomenon at midlatitudes, originate in the auroral zone as gravity waves. Curiously, however, the latter do not seem to be related to magnetic activity. These atmospheric waves are common at high latitudes (Bristow and Greenwald, 1996; Bristow et al., 1996), and we argue here that, as they propagate to lower latitudes, Joule damping reduces the gravity wave spectrum to waves suffering the weakest damping. The direction of weakest damping corresponds to the direction predicted by the Perkins instability (Perkins, 1973) for nighttime MSTIDs. The daytime features reported by Ogawa et al. (2009) are very likely due to classical gravity wave interaction with the F-region ionosphere.
\end{abstract}

Keywords. Ionosphere (Mid-latitude ionosphere; Plasma waves and instabilities; Wave propagation)

\section{Introduction}

Mesoscale Traveling Ionospheric Disturbances (MSTIDs) have been studied for many decades. They were first discovered using ionosondes (e.g., Bowman, 1985, 1989). Breakthroughs came with coherent scatter radar (Fukao et al., 1991; Kelley and Fukao, 1991) and airglow instrumentation (Mendillo et al., 1997; Garcia et al., 2000a, b). Five examples of midlatitude airglow features are shown in Fig. 1 and are compared with airglow from the magnetic equator at the bottom right. The striking difference is that the midlatitude features are not aligned with the magnetic meridian and do not move eastward as the equatorial features do, but rather propagate southwest in the Northern Hemisphere and northwest in the Southern Hemisphere.

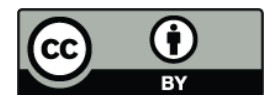

Correspondence to: M. C. Kelley (mck13@ cornell.edu)
The use of the term "mesoscale" refers to the typical wavelengths in the range of $300-1000 \mathrm{~km}$. Conversely, large-scale TIDs travel equatorward out of the auroral oval, have typical wavelengths over $1000 \mathrm{~km}$, and travel much faster, up to a factor of ten faster, than MSTIDs. Use of the term "TID" leads one to assume that the features are caused by the interaction of gravity waves with ionospheric plasma. In the case of large-scale TIDs, this interaction is caused by the meridional wind moving plasma up and down the inclined magnetic field. The resulting altitude change affects the recombination rate and hence the plasma density. Ogawa et al. (2009) report an example of a daytime MSTID that has similar characteristics to large-scale TIDs but does not have F-region irregularities or the E-region effects that characterize nighttime MSTIDs and is likely due to classical gravity wave interaction with the ionosphere. In the remainder of this paper, the term "MSTIDs" refers exclusively to nighttime MSTIDs. MSTIDs are much more complicated and more interesting. In particular, the MU radar (Fukao et al., 1991; Kelley and Fukao, 1991) showed that MSTIDs were electrified with internal velocities over $200 \mathrm{~m} \mathrm{~s}^{-1}$. This result was obtained in the coherent scatter mode that uses field-aligned irregularities as targets. About ten years earlier, Behnke (1979) used the Arecibo incoherent scatter radar mode and published the first experimental paper on the topic. In one event, he found over $400 \mathrm{~m} \mathrm{~s}^{-1}$ internal velocities.

With the development of image intensifiers and then CCD cameras, it became possible to take pictures of these structures, a major breakthrough since previously, only onedimensional measurements were possible. The airglow data revealed the three-dimensional structure of the waves and their phase velocity: southwest in the Northern Hemisphere and northwest in the south. Other data sets became available as interest grew. Jacobson et al. (1995) used the Very Large Array to study hundreds of events and verified the airglow results. Multi-experimental studies showed that the waves are electrostatic, that is, $\boldsymbol{k} \cdot \boldsymbol{E}=0$ (Saito et al., 1998), and that identical structures exist (Otsuka et al., 2004) in the conjugate hemisphere, a clear indication of their electrical nature.

Published by Copernicus Publications on behalf of the European Geosciences Union. 

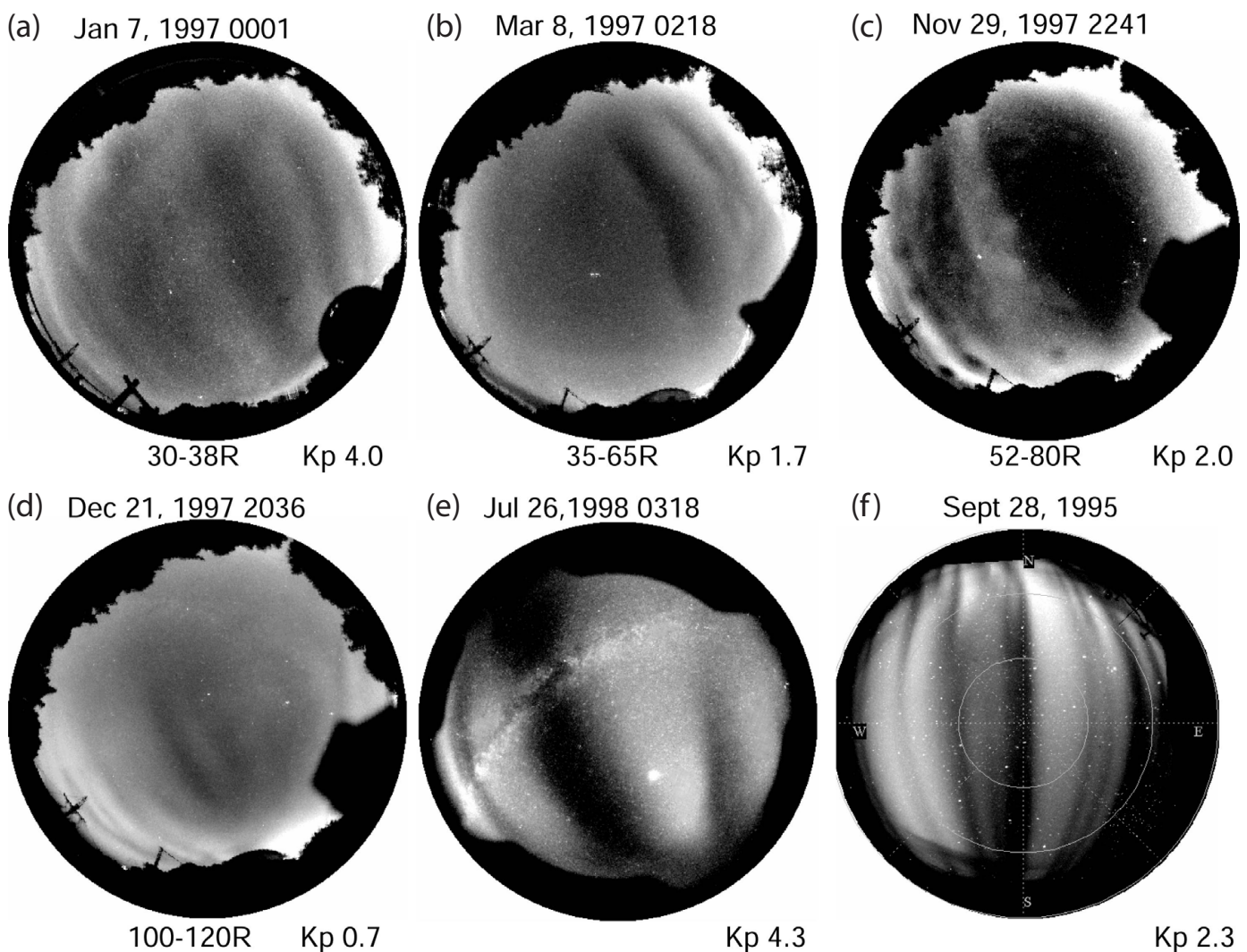

$\mathrm{Kp} 4.3$

$\mathrm{Kp} 2.3$

Fig. 1. Summary of events observed from Puerto Rico (top row and bottom left), Hawaii (bottom middle), and Christmas Island (bottom right, courtesy of M. Taylor). Each image has been standardized so that north is at the top and east is to the right (after Kelley et al., 2002, reproduced with permission of Elsevier).

Haldoupis et al. (2003) and Kelley et al. (2003) showed experimentally that the E- and F-regions are closely coupled in this process.

Theoretical work has lagged far behind experiments. Perkins (1973) predicted a plasma instability with the correct $\boldsymbol{k}$ vector, albeit with the wrong sign. The growth rate of the Perkins process is very low, however, and the waves cannot possibly grow from thermal noise. A few nonlinear calculations have shown that, if significantly seeded, waves will grow (Huang et al., 1994; Kelley and Miller, 1997), but the $\boldsymbol{k}$ vector sign problem remains. Recently, three-dimensional simulations were made (Yokoyama et al., 2008, 2009) and coupling between the E- and F-layer instabilities was explored (Cosgrove and Tsunoda, 2004; Cosgrove et al., 2004). Kelley and Miller (1997) posited a new type of wave, an electrobuoyancy wave, to which we return later. There are seasonal and longitudinal effects on MSTIDs (Kotake et al., 2006) that are beyond the scope of this paper. We suspect that sporadic E-layers play a role (Otsuka et al., 2007) as well as the ratio of F- to E-region conductivities. Gravity waves in the E-region may also play a role but were not reported by Ogawa et al. (2009).
We do not know the source of nighttime MSTIDs. However, a major breakthrough occurred recently when Ogawa et al. (2009) used a Superdarn-type radar to show that MSTIDs seem to be related to gravity waves generated at high latitudes. However, they found no relationship with magnetic activity, that is, the gravity wave amplitude and occurrence rate did not depend on Kp. However, Codrescu et al. (2000) have shown that small-scale electric field fluctuations can lead to Joule heating effects, even at low Kp. Alternatively, the in situ generation of gravity waves discussed by Vadas et al. (2003) and Vadas and Liu (2009) may be the source.

In summary, these gravity waves/MSTIDS waves exhibit:

1. Typical horizontal scales of $500 \mathrm{~km}$.

2. Typical periods of an hour or two.

3. Typical electric fields of $5 \mathrm{mV} \mathrm{m}^{-1}$.

4. Southwest (northwest) phase velocities in the Northern (Southern) Hemisphere of $50-150 \mathrm{~m} \mathrm{~s}^{-1}$.

5. Origin at high latitudes but no dependence on magnetic activity.

Here we propose an explanation. 


\section{A background discussion of the Perkins instability}

Perkins created a midlatitude ionospheric model that circumvented many complexities of that region. He created a fieldline-integrated ionosphere that replaced reality with a "single particle or ping-pong ball model" of the ionosphere (Kelley, 2009). In this model, the pressure gradient forces disappear, as does production (nighttime) and loss (high altitude). Gravity, electric field, and wind-driven currents are left to create instability. He found that, under certain conditions, a preexistent irregularity will grow with wavefronts oriented from NW to SE in the Northern Hemisphere.

Instabilities are typically analyzed around an initial stability that may or not be unstable. Perkins noticed that, with an eastward electric field or a southward neutral wind, the ionosphere would rise to a height at which the downward plasma drift due to gravity was balanced by the uplift due to the $\boldsymbol{E} \times \boldsymbol{B}$ drift or the southward wind. However, he showed that the equilibrium is unstable if there is a northward electric field or an eastward wind with a very low growth rate. In addition, if, during evening conditions, these waves were somehow created, they would travel toward the east, not the west (Garcia et al., 2000a, b; Kelley, 2009). These issues are hugely problematic for the pure Perkins instability.

We argue below that it is not the instability that matters but the lack of wave damping for waves that arise as neutral disturbances at high latitudes, called travelling atmospheric disturbances (TADS), and interact with the midlatitude ionosphere to create MSTIDs.

\section{On the origin of MSTIDs}

Chisham et al. (2007) summarized the SUPERDARN observations of many phenomena, including their very common detection of gravity waves at high latitudes. These waves occurred at all levels of magnetic activity, as was also reported later by Ogawa et al. (2009). This notion is supported by satellite data showing that plasma irregularities are present $100 \%$ of the time above $55^{\circ}$ latitude (Kelley and Mozer, 1972).

We do not attempt to quantitatively explain these highlatitude waves but suggest that highly structured Joule heating is a likely source. Kelley and Mozer (1972) detected irregularities through their electric field measurements and it is clear that $d E \sim E$, indicating that the medium-scale Joule heating equals the large-scale heating. Thus, gravity waves may be generated in situ.

We argue here that once these gravity waves are generated more or less isotropically, the mechanism described by Kelley and Miller (1997) will take over. They set up a two-dimensional numerical simulation of the same equations used by Perkins. The equilibrium F-layer height was maintained by an eastward electric field (or a southward neutral wind) acting against gravity. Instability occurs when a north- ward electric field or an eastward wind is applied. Huang et al. (1994) published the results of a similar numerical simulation of the Perkins instability and Yokoyama et al. (2008, 2009) extended the theory to three dimensions.

As shown in Fig. 2, if a random set of fluctuations are input to the midlatitude ionosphere with conditions conducive to the Perkins instability, they all damp out except those in the preferred Perkins direction. Similar results were found by Yokoyama et al. (2008, 2009). Following the experimental results of Ogawa et al. (2009), we believe this process becomes important only when the gravity waves propagate into regions with a finite dip angle; that is, beginning first as gravity waves, they are then converted to electrified MSTIDs at midlatitudes.

Returning to Fig. 2, after one hour, the only surviving wavefronts are aligned northwest to southeast in the Northern Hemisphere. Kelley and Miller (1997) explained this result by pointing out that the Joule heating is smallest for these wavenumbers since, in this direction, the electrical current caused by the perturbation neutral wind in the wave is almost cancelled by the polarization electric field-driven current in the wave. That is, we argue that the ion velocity $\left(\boldsymbol{E} \times \boldsymbol{B} / B^{2}\right)$ equals the wind speed in the wave. Using the DE-2 satellite, Saito et al. (1998) showed that the perturbation $\delta \boldsymbol{E} \times \boldsymbol{B}$ drift is parallel to the MSTIDs wavenumber. In this case, there is little or no $\boldsymbol{J} \times \boldsymbol{B}$ forcing or Joule heating associated with the wave, and the damping is weak.

We now check to see if Joule damping is more important than viscous damping. Ever since the pioneering work of Hines (e.g., Hines, 1974), the issue of whether viscous or Joule damping dominates gravity wave decay has been debated. Experiments indicate that short, vertical wavelength gravity waves are damped by viscosity in the lower thermosphere. Thus, the vertical wavelength of gravity waves that reach the thermosphere (Djuth et al., 1997) must be large and hence the viscosity caused by the vertical spatial derivative is not important for waves that actually reach the thermosphere.

We now consider the damping mechanism of gravity waves caused by horizontal variations in their perturbation quantities. The two damping terms to be compared are the $\boldsymbol{J} \times \boldsymbol{B}$ force and the viscous force in the Navier-Stokes equation (Kelley, 2009). We now solve for the critical scale, $L_{\mathrm{c}}$, such that for horizontal wavelengths larger than $L_{\mathrm{c}}$, the $\boldsymbol{J} \times \boldsymbol{B}$ term dominates viscosity. For arbitrary propagation directions, the current associated with the neutral wind, $\boldsymbol{J}=s_{\mathrm{P}}(\boldsymbol{U} \times \boldsymbol{B})$, will dissipate the wave energy. However, in the special Perkins direction, a polarization electric field will build up such that the current becomes very small and the damping ceases, as shown by the numerical simulation in Fig. 2.

In an ionized gas, ignoring the Coriolis force, the NavierStokes equation is

$\rho d U / d t=-\nabla p+\rho g+\eta \nabla^{2} U+\boldsymbol{J} \times \boldsymbol{B}$ 

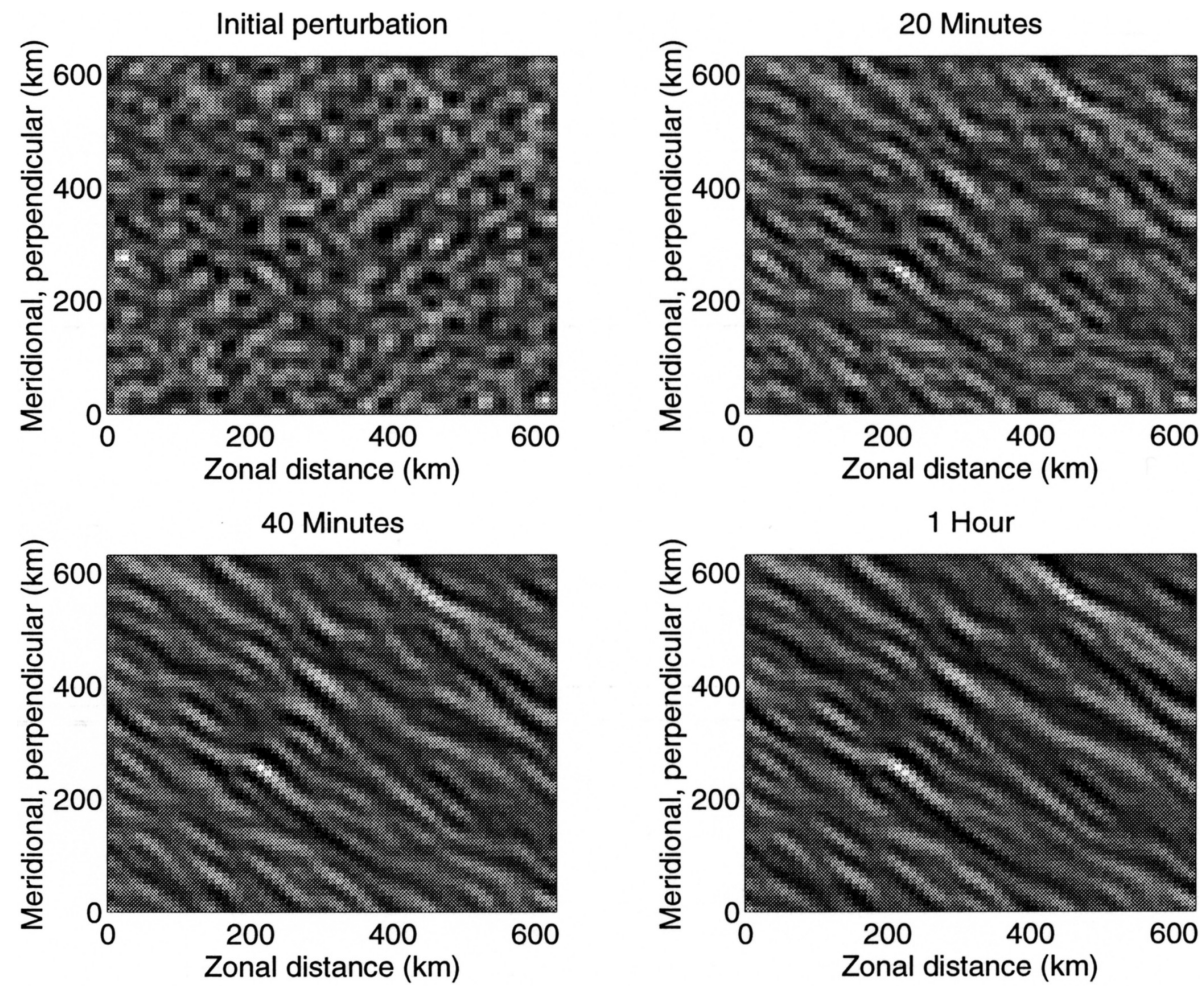

Fig. 2. Azimuthal filtering imposed by the ionospheric electric field response on randomly excited perturbations (after Kelley and Miller, 1997, reproduced with permission of the American Geophysical Union).

where $\eta$ is the viscosity coefficient and the other symbols are traditional. To determine this critical scale, we equate the Joule forcing term $\left[\sigma_{\mathrm{P}}(\boldsymbol{U} \times \boldsymbol{B}) \times \boldsymbol{B}\right]$ to viscous forcing.

We now compare the last two terms by setting them equal and solving for $L_{\mathrm{c}}$,

$\sigma_{\mathrm{P}} U B^{2}=\eta U / L_{\mathrm{c}}^{2}$

where $\sigma_{\mathrm{P}}=n e^{2} v_{\text {in }} / M \Omega^{2}, U$ is the gravity wave perturbation wind speed perpendicular to $\boldsymbol{B}, v_{\text {in }}$ is the ion neutral collision frequency, and $\Omega$ is the ion gyrofrequency. Solving this equation for an $L_{\mathrm{c}}$ yields,

$$
\left(L_{\mathrm{c}}\right)^{2}=\eta / \rho_{i} v_{\text {in }}
$$

where $\rho_{i}$ is the mass density of the plasma. Using neutral parameters from MSIS, a plasma density of $10^{11} \mathrm{~m}^{-3}$, a collision frequency of $1 \mathrm{~s}^{-1}$, and the viscosity coefficient of $3 \times 10^{-6} \mathrm{~kg} \mathrm{~ms}^{-1}$ at $1000 \mathrm{~K}$ from Banks and Kockarts (1973), we find in the thermosphere that $L_{\mathrm{c}}=85 \mathrm{~km}$. This result implies that, for MSTIDs, the Joule forcing dominates and causes waves in all but the Perkins direction to die out.

Recently, Yokoyama et al. (2009) and Yokoyama and Hysell (2010) extended their simulations of E- and F-region coupling in three dimensions. It is clear that the F-region Perkins growth rate is considerably enhanced by this coupling. What is not clear is how the scale sizes in the two regions can be reconciled with each other, since the MSTID scale is many hundreds of kilometers but even the scale of Eregion patches is much smaller; the separation of Q-P echoing regions is smaller still. We suggest here that gravity waves originating in the auroral zone and propagating southwest survive to midlatitudes against viscous and Joule damping and then are amplified via the E- and F-region coupling described by Cosgrove and Tsunoda (2004) and Cosgrove et al. (2004) and simulated by Yokoyama and Hysell (2010).

\section{Conclusions}

We argue that the ubiquity of gravity waves at high latitudes is the source of MSTIDs. As described by Kelley and Miller (1997), all of these waves will be damped at midlatitudes except for those travelling in the Perkins direction, the direction with a minimum in Joule damping. Consequently, these waves can travel thousands of kilometers. Once at midlatitudes, the E- and F-region coupling effect amplifies them and they become MSTIDs.

Acknowledgements. This research was supported by the Atmospheric Science Section of the National Science Foundation under grant ATM-0551107.

Topical Editor K. Kauristie thanks M. Pinnock and two other anonymous referees for their help in evaluating this paper. 


\section{References}

Banks, P. M. and Kockarts, G.: Aeronomy, Parts A and B, Academic Press, New York, 1973.

Behnke, R. A.: F layer height bands in the nocturnal ionosphere over Arecibo, J. Geophys. Res., 84(3), 974-978, 1979.

Bowman, G. G.: Some aspects of mid-latitude spread-E , $_{S}$ and its relationship with spread-F, Planet. Space Sci., 33, 1081-1089, 1985.

Bowman, G. G.: Quasi-periodic scintillations at mid-latitudes and their possible association with ionospheric sporadic-E structures, Ann. Geophys., 7, 259-267, 1989.

Bristow, W. A. and Greenwald, R. A.: Multiradar observations of medium-scale acoustic gravity waves using the Super Dual Auroral Radar Network, J. Geophys. Res., 101, 24499-24511, 1996.

Bristow, W. A., Greenwald, R. A., and Villain, J. P.: On the seasonal dependence of medium-scale atmospheric gravity waves in the upper atmosphere at high latitudes, J. Geophys. Res., 101, 15685-15699, 1996.

Chisham, G., Lester, M., Milan, S. E., Freeman, M. P., Bristow, W. A., Grocott, A., McWilliams, K. A., Ruohoniemi, J. M., Yeoman, T. K., Dyson, P. L., Greenwald, R. A., Kikuchi, T., Pinnock, M., Rash, J. P. S., Sato, N., Sofko, G. J., Villain, J.-P., and Walker, A. D. M.: A decade of the Super Dual Auroral Radar Network (SuperDARN): Scientific achievements, new techniques and future directions, Surv. Geophys., 28(1), 33-109, doi:10.1007/s10712007-9017-8, 2007.

Codrescu, M. V., Fuller-Rowell, T. J., Foster, J. C., Holt, J. M., and Cariglia, S. J.: Electric field variability associated with the Millstone Hill electric field model, J. Geophys. Res., 105(A3), 5265-5273, doi:10.1029/1999JA900463, 2000.

Cosgrove, R. B. and Tsunoda, R. T.: Instability of the E-F coupled nighttime midlatitude ionosphere, J. Geophys. Res., 109(A4), A04305, doi:10.1029/2003JA010243, 2004.

Cosgrove, R. B., Tsunoda, R. T., Fukao, S., and Yamamoto, M.: Coupling of the Perkins instability and the sporadic E layer instability derived from physical arguments, J. Geophys. Res., 109, A06301, doi:10.1029/2003JA010295, 2004.

Djuth, F., Sulzer, M., Elder, J., and Wickwar, V.: High-resolution studies of atmosphere-ionosphere coupling at Arecibo Observatory, Puerto Rico, Radio Sci., 32(6), 2321-2344, 1997.

Fukao, S., Kelley, M. C., Shirakawa, T., Takami, T., Yamamoto, M., Tsuda, T., and Kato, S.: Turbulence upwelling of the midlatitude ionosphere, 1. Observational results by the MU radar, J. Geophys. Res., 96, 3725-3746, 1991.

Garcia, F. J., Kelley, M. C., Makela, J. J., and Huang, C.-S.: Airglow observations of mesoscale low-velocity traveling ionospheric disturbances at midlatitudes, J. Geophys. Res., 105(A8), 18407-18415, 2000a.

Garcia, F. J., Kelley, M. C., Makela, J. J., Sultan, P. J., Pi, X., and Musman, S.: Mesoscale structure of the midlatitude ionosphere during high geomagnetic activity: Airglow and GPS observations, J. Geophys. Res., 105(A8), 18417-18427, 2000 b.

Haldoupis, C., Kelley, M. C., Hussey, G. C., and Shalimov, S.: Role of unstable sporadic-E layers in the generation of midlatitude spread F, J. Geophys. Res., 108(A12), 1446, doi:10.1029/2003JA009956, 2003.

Hines, C. O.: The Upper Atmosphere in Motion: A Selection of Papers with Annotation. Geophys. Monogr., 18, American Geophysical Union, Washington, D.C., 1974.
Huang, C. S., Miller, C. A., and Kelley, M. C.: Basic properties and gravity wave initiation of the midlatitude $\mathrm{F}$ region instability, Radio Sci., 29(1), 395-405, 1994.

Jacobson, A. R., Carlos, R. C., Massey, R. S., and Wu, G.: Observations of traveling ionospheric disturbances with a satellitebeacon radio interferometer: Seasonal and local time behavior, J. Geophys. Res., 100, 1653-1665, 1995.

Kelley, M. C.: The Earth's Ionosphere: Plasma Physics and Electrodynamics, 2nd ed., International Geophysics Series, vol. 96, Elsevier, London, 2009.

Kelley, M. C. and Fukao, S.: Turbulent upwelling of the midlatitude ionosphere: 2. Theoretical framework, J. Geophys. Res., 96, 3747-3753, 1991.

Kelley, M. C. and Miller, C. A.: Electrodynamics of midlatitude spread F, 3, Electrohydrodynamic waves? A new look at the role of electric fields in thermospheric wave dynamics, J. Geophys. Res., 102(A6), 11539-11547, 1997.

Kelley, M. C. and Mozer, F. S.: A satellite survey of vector electric fields in the ionosphere at frequencies of 10 to 500 Hertz, 1. Isotropic, high latitude electrostatic emissions, J. Geophys. Res., 77, 4158-4173, 1972.

Kelley, M. C., Makela, J. J., and Saito, A.: The mid-latitude F region at the mesoscale: Some progress at last, J. Atmos. Solar-Terr. Phys., 64, 1525-1529, 2002.

Kelley, M. C., Haldoupis, C., Nicolls, M. J., Makela, J. J., Belehaki, A., Shalimov, S., and Wong, V. K.: Case studies of coupling between the $\mathrm{E}$ and $\mathrm{F}$ regions during unstable sporadic-E conditions, J. Geophys. Res., 108(A12), 1447, doi:10.1029/2003JA009955, 2003.

Kotake, N., Otsuka, Y., Tsugawa, T., Ogawa, T., and Saito, A.: Climatological study of GPS total electron content variations caused by medium-scale traveling ionospheric disturbances, J. Geophys. Res., 111, A04306, doi:10.1029/2005JA011418, 2006.

Mendillo, M., Baumgardner, J., Nottingham, D., Aarons, J., Reinisch, B., Scali, J., and Kelley, M.: Investigations of thermospheric-ionospheric dynamics with $6300-\AA \AA$ images from the Arecibo Observatory, J. Geophys. Res., 102, 7331-7343, 1997.

Ogawa, T., Nishitani, N., Otsuka, Y., Shiokawa, K., Tsugawa, T., and Hosokawa, K.: Medium-scale traveling ionospheric disturbances observed with the SuperDARN Hokkaido radar, allsky imager, and GPS network and their relation to concurrent sporadic-E irregularities, J. Geophys. Res., 114, A03316, doi:10.1029/2008JA013893, 2009.

Otsuka, Y., Shiokawa, K., Ogawa, T., and Wilkinson, P.: Geomagnetic conjugate observations of medium-scale traveling ionospheric disturbances at midlatitude using allsky airglow imagers, Geophys. Res. Lett., 31, L15803, doi:10.1029/2004GL020262, 2004.

Otsuka, Y., Onoma, F., Shiokawa, K., Ogawa, T., Yamamoto, M., and Fukao, S.: Simultaneous observations of nighttime medium-scale traveling ionospheric disturbances and $\mathrm{E}$ region field-aligned irregularities at midlatitude, J. Geophys. Res., 112, A06317, doi:10.1029/2005JA011548, 2007.

Perkins, F. W.: Spread F and ionospheric currents, J. Geophys. Res., 78, 218-226, 1973.

Saito, A., Iyemori, T., Blomberg, L. G., Yamamoto, M., and Takeda, M.: Conjugate observations of the mid-latitude electric field fluctuations with the MU radar and the Freja satellite, J. Atmos. 
Solar-Terr. Phys., 60(1), 129-140, 1998.

Vadas, S. L. and Liu, H.: Generation of large-scale gravity waves and neutral winds in the thermosphere from the dissipation of convectively generated gravity waves, J. Geophys. Res., 114, A10310, doi:10.1029/2009JA014108, 2009.

Vadas, S. L., Fritts, D. C., and Alexander, M. J.: Mechanism for the generation of secondary waves in wave breaking regions, J. Atmos. Sci., 60, 194-214, 2003.

Yokoyama, T. and Hysell, D. L.: A new midlatitude ionosphere electrodynamics coupling model (MIECO): Latitudinal dependence and propagation of medium-scale traveling ionospheric disturbances, Geophys. Res. Lett., 37, L08105, doi:10.1029/2010GL042598, 2010.
Yokoyama, T., Otsuka, Y., Ogawa, T., Yamamoto, M., and Hysell, D. L.: First three-dimensional simulation of the Perkins instability in the nighttime midlatitude ionosphere, Geophys. Res. Lett., 35, L03101, doi:10.1029/2007GL032496, 2008.

Yokoyama, T., Hysell, D. L., Otsuka, Y., and Yamamoto, M.: Threedimensional simulation of the coupled Perkins and $E_{S}$-layer instabilities in the nighttime midlatitude ionosphere, J. Geophys. Res., 114, A03308, doi:10.1029/2008JA013789, 2009. 